\title{
Semantic relationism, belief reports and contradiction
}

\author{
Paolo Bonardi
}

Published online: 11 October 2012

(C) Springer Science+Business Media B.V. 2012

\begin{abstract}
In his book Semantic Relationism, Kit Fine propounds an original and sophisticated semantic theory called 'semantic relationism' or 'relational semantics', whose peculiarity is the enrichment of Kaplan's, Salmon's and Soames' Russellian semantics (more specifically, the semantic content of simple sentences and the truth-conditions of belief reports) with coordination, "the very strongest relation of synonymy or being semantically the same". In this paper, my goal is to shed light on an undesirable result of semantic relationism: a report like "Tom believes that Cicero is bald and Tom does not believe that Tully is bald" is correct according to Fine's provided truth-conditions of belief reports, but its semantic content is (very likely) a contradiction. As I will argue in the paper, even the resort to the notion of token proposition, introduced in Fine's recent article "Comments on Scott Soames' 'Coordination Problems", does not suffice to convincingly eliminate the contradiction; moreover, it raises new difficulties.
\end{abstract}

Keywords Semantic relationism - Coordination - Token propositions · Belief reports · Contradiction

\section{Preamble}

In his book Semantic Relationism, Fine propounds an original and sophisticated semantic theory called 'semantic relationism' or 'relational semantics', whose peculiarity is the enrichment of Kaplan's, Salmon's and Soames' Russellian semantics (more specifically, the semantic content of simple sentences and the

\footnotetext{
P. Bonardi $(\bowtie)$

Department of Philosophy, Université de Genève, Geneva, Switzerland

e-mail: pbonardi1@gmail.com

P. Bonardi

Department of Philosophy and Religion, Rutgers University, Camden, NJ, USA
} 
truth-conditions of belief reports) with coordination, "the very strongest relation of synonymy or being semantically the same" (2007: 5). By performing this manoeuvre, Fine aims

to defend a [Russellian] position within the philosophy of language. For coordination can do much of the work of sense; and, by adopting a relationalist view of coordination, the [Russellian philosopher] can secure many of the advantages of the Fregean position without being committed to the existence of sense. (2007: 5)

In this paper, my goal is to shed light on an undesirable result of semantic relationism: a report like "Tom believes that Cicero is bald and Tom does not believe that Tully is bald" is correct according to Fine's provided truth-conditions of belief reports, but its semantic content is (very likely) a contradiction. As I will argue in the paper, even the resort to the notion of token proposition, introduced in Fine's recent article "Comments on Scott Soames' 'Coordination Problems', does not suffice to convincingly eliminate the contradiction; moreover, it raises new difficulties.

My criticisms of Fine presuppose a presentation of his semantics, starting from the key notion of coordination.

\section{Coordination}

Fine (2007: 39-40) characterizes coordination among proper names as follows.

Characterisation 1: Two co-referential token names (viz. two tokens of the same name or two tokens of different but co-referential names) in a piece of discourse are positively coordinated if and only if they represent their referent as the same. This is in turn the case if and only if no (sincere, reflexive and non-reticent) hearer/speaker who understands the discourse can sensibly raise the question of whether their referent is the same. Two co-referential token names in a piece of discourse are negatively coordinated if and only if they are not positively coordinated. ${ }^{1}$

For example, suppose that you say (1) and (2). Independently of your knowing or explicitly declaring that Cicero is Tully, a hearer who understands your piece of discourse can sensibly raise the question of whether the names 'Cicero' and 'Tully' within it co-refer. Therefore, according to Characterisation 1, these names are negatively coordinated.

(1) Cicero was an orator.

(2) Tully wrote the De Fato.

On the other hand, suppose that you say "Cicero was an orator and the same Cicero was astute" or "Cicero was an orator and Cicero was also astute". The

\footnotetext{
${ }^{1}$ Hoping to correctly understand the notion of coordination, I have added in Characterisation 1 the second 'only if' to Fine's (2007: 39-40) original formulation.
} 
presence of the linguistic particle 'same' or 'also' in this statement makes evident your intention to use the two tokens of 'Cicero' in the same way (viz. as co-referring tokens). Thus, no hearer who understands your piece of discourse would sensibly question whether these tokens co-refer, to the effect that they will be positively coordinated, according to Characterisation 1. It should be noted that two tokens of 'Cicero' are positively coordinated also when the presuppositions of the discourse make clear that they are used in the same way; in this case, the presence in your statement of the particle 'same' or 'also' is superfluous in order to yield positive coordination. $^{2}$

Besides Characterisation 1, Fine (2007: 43-50) also offers a second more technical characterisation of the notion of coordination among names. Examination of it might help the reader to better understand the intuitive Characterisation 1 .

Characterisation 2: Two co-referential token names in a piece of discourse are positively (i.e. non-negatively) coordinated if and only if they represent their referent as the same. This is in turn the case if and only if they strictly co-refer, i.e. if and only if it is a semantic requirement that they co-refer.

A semantic requirement is a semantic fact in a narrow sense. A fact, belonging to the semantics of a given language $L$, is semantic in a narrow sense if "any rational and reflective individual who understands $L$ is [...] in a position to know that the fact obtains" (2007: 60). For example, the fact that the names 'Cicero' and 'Tully' co-refer is semantic in a broad but not narrow sense, because an English

[...] speaker may know that 'Cicero' refers to a particular person and know that 'Tully' refers to a particular person without being in a position to know that they are coreferential. (2007: 46)

Since the fact that 'Cicero' and 'Tully' co-refer is not semantic in a narrow sense then, according to Characterisation 2, 'Cicero' and 'Tully' do not strictly co-refer, with the result that they will be negatively coordinated. Instead, the two tokens of the name 'Cicero' within the statement "Cicero was an orator and [the same] Cicero was astute" are positively coordinated: the fact that these tokens co-refer is available to all English speakers and therefore it is semantic in a narrow sense.

Besides cases of positive and negative coordination, in Fine's semantics there is also the possibility of having cases of uncoordination (i.e. neither positive nor negative coordination). Suppose, for instance, that the statements "Cicero was an orator" and "Cicero was astute" belong to different pieces of discourse. If so, we cannot establish whether the two tokens of the name 'Cicero' in these statements are positively or negatively coordinated: coordination is defined relative to one given piece of discourse (see Characterisations 1 and 2), having its own presuppositions and being produced by a speaker who has particular referential intentions.

\footnotetext{
${ }^{2}$ On the contrary, if neither your statement contains particles like 'same', 'also' nor do the presuppositions of the discourse make clear your intention to co-refer, a hearer of the statement "Cicero was an orator and Cicero was astute" (in particular, one who knows that two famous Ciceros have existed, the Roman orator and the German spy) may sensibly question whether the two tokens of 'Cicero' in this statement co-refer, consequently making coordination between them negative.
} 
Hence, 'Cicero' and 'Tully' as belonging to separate/isolated statements are uncoordinated (or not coordinated). ${ }^{3}$

\section{Relational semantics of simple sentences}

Having characterized the notion of coordination, let us now see what roles this notion plays in Fine's semantics. As a first important role, coordination contributes to the semantic content of simple sentences/statements in the following way. ${ }^{4}$ Consider a simple sentence containing two co-referring terms. Fine (2007, Ch. 2, $\S \mathrm{F})$ distinguishes between two semantic contents of this sentence: its primary content, which is simply the Russellian proposition expressed by it; and its secondary content, i.e. the coordinated proposition formed by its primary content plus the relation of (positive or negative) coordination holding between the two aforementioned co-referring terms.

For example, the primary content of sentence (3) is the Russellian proposition (3p), while its secondary content is the negatively coordinated proposition $\left(3 \mathrm{p}^{*}\right)$, formed by ( $3 \mathrm{p})$ plus the relation of negative coordination, $C^{-}$, holding between the names 'Cicero' and 'Tully' in (3).

(3) Cicero admires Tully

(3p) $<$ Cicero, Admiration, Cicero $>$

$\left(3 \mathrm{p}^{*}\right)<<$ Cicero, Admiration, Cicero $>, C^{-}>$

The primary content of statement (4) is the same as that of (3), i.e. proposition (3p), whereas its secondary content differs: it is the positively coordinated proposition $\left(4 \mathrm{p}^{*}\right)$, formed by $(3 \mathrm{p})$ plus the relation of positive coordination, $C^{+}$, holding between the two tokens of the name 'Cicero' (used in the same way) within statement (4).

(4) Cicero admires [the same] Cicero

$\left(4 p^{*}\right) \quad<<$ Cicero, Admiration, Cicero $>, C^{+}>$

A simple sentence like (1) not containing co-referring names has solely primary content, i.e. (1p), if it belongs to a piece of discourse which does not include, besides 'Cicero' in (1), other terms referring to the individual Cicero.

(1p) <Cicero, Having been an orator $>$

\footnotetext{
${ }_{3}^{3}$ Although Fine does not explicitly mention the coordination/uncoordination distinction (he generally uses the terms 'coordination' and 'uncoordination' as synonyms of 'positive' and 'negative coordination' respectively), such a distinction-I think-implicitly emerges e.g. in the passages of Semantic Relationism (2007: 93, 101) where, talking of the reports "Peter believes that Paderewski is musical" and "Peter believes that Paderewski is not musical", he distinguishes between the case in which these reports are considered individually, in isolation (i.e. as belonging to different pieces of discourse, to the effect that the two tokens of 'Paderewski' within them will be uncoordinated) and the case in which they are taken as one pair of reports (and therefore as belonging to the same piece of discourse, with the result that the two tokens of 'Paderewski' within such reports will be coordinated).

${ }^{4}$ By simple sentence I mean "a sentence that does not contain any quotational, psychological, or other obviously non-extensional linguistic contexts" (Braun and Saul 2002: 2).
} 
On the other hand, if (1) belongs to a piece of discourse which includes another sentence about Cicero, e.g. (2), then the pair of sentences (1), (2) will express the negatively coordinated pair of propositions (5), formed by the pair of Russellian propositions expressed by (1) and (2) plus the relation of negative coordination between the names 'Cicero' and 'Tully' within these sentences.

(5) $<<<$ Cicero, Having been an orator $>,<$ Cicero, Having written the De Fato $>>, C^{-}>$

\section{Relational semantics of belief reports}

Another important role coordination plays in Fine's semantics is its contribution to the Sufficient Conditions for the Correctness (SCC) of belief reports. Call the conjunction of two belief reports composite report. Fine (2007: 102-5) distinguishes among three different readings of a composite report containing two co-referring terms: pure de re, weak de dicto and strict de dicto readings.

\subsection{Sufficient conditions for the correctness of these three readings}

Suppose that a subject $S$ sincerely, on reflection and competently makes the statements ' $a_{1}$ is $F$ ' and ' $b_{1}$ is $G$ ', where the token terms ' $a_{1}$ ' and ' $b_{1}$ ' co-refer. The pure de re reading of the composite report ' $S$ believes that $a_{2}$ is $F$ and $S$ believes that $b_{2}$ is $G$ ' is correct if condition (i) (below) is satisfied. Its weak de dicto reading is correct if conditions (i) and (ii) are jointly satisfied. Finally, its strict de dicto reading is correct if conditions (i), (ii) and (iii) are jointly satisfied.

(i) The (token) terms ' $a_{2}$ ' and ' $b_{2}$ ' have the same referent as respectively ' $a_{1}$ ' and ' $b_{1}$ '.

(ii) The two pairs of terms ' $a_{2}$ ', ' $b_{2}$ ' and ' $a_{1}$ ', ' $b_{1}$ ' are co-coordinated, i.e. they are coordinated in the same way (both positively or both negatively).

(iii) The two pairs of terms ' $a_{2}$ ', ' $b_{2}$ ' and ' $a_{1}$ ', ' $b_{1}$ ' are cross-coordinated, i.e. ' $a_{2}$ ' is positively coordinated with ' $a_{1}$ ' and ' $b_{2}$ ' is positively coordinated with ' $b_{1}$ '.

For example, suppose that Tom, who fails to realize that Cicero is Tully, sincerely asserts "Cicero is bald" and "Tully is not bald". Now, consider the following composite reports:

(6) Tom believes that Cicero is bald and Tom believes that Tully is not bald;

(7) Tom believes that Tully is bald and Tom believes that Cicero is not bald;

(8) Tom believes that Cicero is bald and Tom believes that [the same] Cicero is not bald.

\footnotetext{
5 The notions of coordinated proposition and coordinated pair of propositions are more sophisticated than presented here: they involve a coordination-scheme (2007: 55-56) rather than a single coordination link. However, for the objectives of this paper, the proposed characterisations of these notions suffice.
} 
The (pure) de re reading of reports (6)-(8) is correct, since condition (i) is satisfied: the pairs of token names of Cicero contained in each report above have the same referent as the token names 'Cicero' and 'Tully' contained in Tom's statements "Cicero is bald" and "Tully is not bald". Incidentally, from the correctness of the de re reading of (6)-(8) it emerges that, within this reading, co-referring names are intersubstitutable salva veritate.

Under their weak de dicto reading, (6) and (7) are correct, since co-coordination, i.e. condition (ii), also holds: the pairs 'Cicero', 'Tully' in (6) and 'Tully', 'Cicero' in (7) are both negatively coordinated, exactly as is the pair 'Cicero', 'Tully' in Tom's statements "Cicero is bald" and "Tully is not bald". On the contrary, there is no reason to take the weak de dicto reading of (8) as correct: the pair of token names (used in the same way) 'Cicero', 'Cicero' in (8), unlike the pair 'Cicero', 'Tully' in Tom's statements, are positively coordinated; therefore, co-coordination does not hold.

As regards, finally, the strict de dicto reading of (6)-(8), only report (6) is correct under this reading, since only in this report cross-coordination, i.e. condition (iii), holds: the token of the name 'Cicero' in (6) is positively coordinated with its token in Tom's statement "Cicero is bald", and positive coordination also holds between the two tokens of 'Tully' in (6) and in Tom's statement "Tully is not bald".

\subsection{Semantic content of the three readings}

Fine does not say in a straightforward manner what the semantic content of a composite report is. Nonetheless, his relational semantics of simple sentences (Sect. 3), the SCC assigned to composite reports (Sect. 4.1), his refusal of Fregean senses and, finally, certain considerations he makes in his book (2007: 77) lead me to the following conclusions.

Since, as we have seen, coordination plays no role within the SCC of the de re reading of a composite report and co-referring names are intersubstitutable salva veritate within this reading, it seems reasonable to claim that the de re reading of a report like (6) or (7) or (8) simply expresses the Russellian proposition (6p).

(6p) $<<$ Tom, B, <Cicero, Baldness $>>$, CONJ, $<$ Tom, B, $<<$ Cicero, Baldness $>, \mathrm{NEG}>>>^{6}$

The SCC of the weak de dicto reading of a composite report crucially involve coordination among terms included in the report; accordingly, this reading should express a coordinated proposition. For example, the weak de dicto reading of (6) or (7) will express the negatively coordinated proposition (6p*), where the relation $C^{-}$ holds between the names 'Cicero' and 'Tully' in either report.

$\left(6 p^{*}\right) \quad<<<$ Tom, B, $<$ Cicero, Baldness $>>$, CONJ, $<$ Tom, B, $<<$ Cicero, Baldness $>, \mathrm{NEG}>>>, C^{-}>$

As regards, finally, the strict de dicto reading of a composite report, we have seen that its SCC involve coordination both among terms included in the report and with

\footnotetext{
${ }^{6}$ CONJ is the relation of Conjunction and NEG is the property of Negation.
} 
terms outside the report which are contained in statements made by the believer. Such a reading will therefore express a kind of proposition more sophisticated than a Russellian or a coordinated proposition, which Fine calls token proposition.

\section{Token propositions}

According to Fine (2007, 54-55), “differences in 'coordination' among names show up as differences in coordination among the objects to which they correspond". For example, negative coordination between the names 'Cicero' and 'Tully' in (3) shows up as negative coordination between the two occurrences of the individual Cicero in $\left(3 \mathrm{p}^{*}\right)$. On the other hand, positive coordination between the two token names 'Cicero' in (4) shows up as positive coordination between the two occurrences of the individual Cicero in $\left(4 \mathrm{p}^{*}\right){ }^{7}$ So, coordination, which is primarily a relation among linguistic expressions, also derivatively holds among the "objects" (viz. occurrences of individuals, of properties, of relations within Russellian propositions) for which these expressions stand. Taking for granted this, let us now define the notion of token individual.

Characterisation 3: A token of an individual is an abstract object identified by a class of occurrences of this individual within a given body of propositions, such that every pair of occurrences belonging to the class is positively coordinated and occurrence positively coordinated with an occurrence in the class is also in the class.

Analogous characterisations can be provided for a token property and a token relation. Finally, a token proposition is defined as a structured proposition whose basic constituents are token individuals, token properties and token relations. ${ }^{8}$

As an example of a token proposition, consider a piece of discourse containing the statements (1), (2) and (6), which expresses the body of Russellian propositions (1p), (2p) and (6p). The token names 'Cicero' and 'Tully' in (6) are negatively coordinated to one another and they are positively coordinated with respectively 'Cicero' in (1) and 'Tully' in (2). Given this, the token individual Cicero will be the abstract object identified by the set of positively coordinated occurrences of Cicero including his occurrence in (1p) and his first occurrence in (6p), while the token individual Tully will be the abstract object identified by the set of positively coordinated occurrences of Cicero including his occurrence in (2p) and his second occurrence in (6p). These two tokens of the individual Cicero enter the token proposition that Tom believes that Cicero is bald and Tom believes that Tully is not bald expressed by the strict de dicto reading of (6), to the effect that Tom will believe and disbelieve (i.e. believe-false), at the strict de dicto level,

\footnotetext{
7 The notion of occurrence of a real individual could perplex the reader. Actually, although it is implausible to maintain that a real individual has multiple occurrences in space-time, there seems to be nothing wrong with the claim that such an individual has multiple occurrences in abstract objects, e.g. in ordered sets or in Russellian propositions.

${ }^{8}$ See Fine (2010: 479-80).
} 
two different token propositions, respectively that Cicero is bald and that Tully is bald.

(2p) <Cicero, Having written the De Fato $>$

\section{True contradictions}

Unlike Salmon and other Russellian philosophers, Fine (2007: 138, n. 4) endorses (at least in the cases which are relevant in this paper) the principle of Negative Disquotation.

Negative Disquotation: If a non-reticent subject $S$ does not have the disposition to sincerely, on reflection and competently assert sentence ' $p$ ', which lacks indexical or pronominal devices or ambiguities, or equivalently if $S$ has such a disposition towards 'I do not believe that $p$ ', then $S$ does not believe that $p{ }^{9}$

It is rather evident that once this principle is accepted, the SCC of composite reports of the form ' $S$ believes that $a_{2}$ is $F$ and $S$ believes that $b_{2}$ is $G$ ' illustrated in Sect. 4.1 can be extended to composite reports (containing negative reports) of the form ' $S$ believes that $a_{2}$ is $F$ and $S$ does not believe that $b_{2}$ is $G$ ', under the assumption that $S$ sincerely, on reflection and competently asserts ' $a_{1}$ is $F$ ' (or equivalently 'I believe that $a_{1}$ is $F$ ') and 'I do not believe that $b_{1}$ is $G$ '.

Now, suppose that Tom, who fails to realize that Cicero is Tully, sincerely asserts "Cicero is bald" and "I do not believe that Tully is bald". Also, consider the composite report

(9) Tom believes that Cicero is bald and Tom does not believe that Tully is bald

According to the SCC extended to composite reports containing negative reports, Fine's three readings of (9) are correct, since conditions (i)-(iii) (Sect. 4.1) are satisfied: (i) the token names 'Cicero' and 'Tully' in (9) have the same referent as the corresponding tokens in Tom's abovementioned statements "Cicero is bald" and "I do not believe that Tully is bald" (co-reference); (ii) the tokens 'Cicero' and 'Tully' in (9) are negatively coordinated, exactly as those in Tom's statements (co-coordination); (iii) the token of 'Cicero' in (9) is positively coordinated with its token in Tom's statement "Cicero is bald", and the token of 'Tully' in (9) is positively coordinated with its token in Tom's statement "I do not believe that Tully is bald" (cross-coordination). From the correctness of these three readings of (9) violations of the principle of non-contradiction follow at the level of their semantic content.

\footnotetext{
9 ' $S$ does not believe that $p$ ' is nothing but the negation of ' $S$ believes that $p$ '.
} 


\subsection{De re and weak de dicto contradictions}

In particular, taking for granted what was stated in Sect. 4.2 about the semantic content of belief reports, the de re and weak de dicto readings of report (9) will respectively express the true self-contradictory propositions $(9 \mathrm{p})$ and $\left(9 \mathrm{p}^{*}\right){ }^{10}$

(9p) $<<$ Tom, B, <Cicero, Baldness $>>$, CONJ, $<<$ Tom, B, $<$ Cicero, Baldness $>>$, NEG $>>$

(9p*) $<<<$ Tom, B, <Cicero, Baldness $>>$, CONJ, $<<$ Tom, B, $<$ Cicero, Baldness $>>, \mathrm{NEG}>>, \mathrm{C}^{-}>$

\subsection{Even strict de dicto contradiction?}

The principle of non-contradiction seems to be safeguarded only at the level of the content of the strict de dicto reading of (9), i.e. at the level of the token proposition that Tom believes that Cicero is bald and Tom does not believe that Tully is bald, provided that the token individuals Cicero and Tully entering this proposition differ.

However, even at the strict de dicto level, a doubt arises. Consider a piece of discourse including report (9) and no term referring to Cicero except for those contained in (9). Since coordination has only been characterized among terms which belong to the same piece of discourse (see Characterisations 1 and 2) and, derivatively, among occurrences of objects within the body of Russellian propositions expressed by such a piece of discourse, the token individual Cicero will be identified, in this case, by the singleton containing the first occurrence of Cicero in (9p), while the token individual Tully will be identified by the singleton containing the second occurrence of Cicero in (9p). Now, if these are the tokens of the individual Cicero entering the token proposition expressed by (9), I wonder how such a proposition could significantly differ from the self-contradictory proposition (9p) - I mean, if the Russellian proposition (9p) is self-contradictory despite its containing two distinct occurrences of Cicero, then the token proposition expressed by (9) should be self-contradictory as well despite its containing the singletons of these occurrences; in other words, it is unclear to me how the move from talking of occurrences to talking of singletons of the very same occurrences would eliminate the contradiction.

\footnotetext{
${ }^{10}$ In response to this objection of mine, Fine has claimed that $(9 \mathrm{p})$ and $\left(9 \mathrm{p}^{*}\right)$ are not genuine contradictions. By contrast, the coordinated proposition formed by (9p) plus positive coordination between the two occurrences of Cicero in $(9 \mathrm{p})$ is genuinely contradictory. This positively coordinated proposition, which e.g. is expressed by the weak de dicto reading of "Tom believes that Cicero is bald and Tom does not believe that [the same] Cicero is bald", is not true under our previous supposition that Tom sincerely asserts "Cicero is bald" and "I do not believe that Tully is bald", since co-coordination does not hold. My point, on the other hand, is that semantic relationism contains violations of the standard principle of non-contradiction; and, undoubtedly, the true propositions (9p) and (9p*) are (or incorporate) standard self-contradictions.
} 


\section{Additional doubts about the notion of a token proposition}

\subsection{Drift towards Fregeanism}

Fine (2007: 5) describes semantic relationism as "an attempt to defend a [Russellian] position within the philosophy of language". On the other hand, once the notion of token proposition is introduced in relational semantics, a cornerstone of Russellianism, viz. the Millian thesis stating that proper names contribute to propositions solely with their referent, is given up in belief reports and in simple sentences (if simple sentences, besides primary and secondary contents, also have tertiary content, i.e. also express a token proposition): as we saw in Sect. 5, within a piece of discourse containing (1), (2) and (6), the (co-referring) names 'Cicero' and 'Tully' contribute to the token proposition expressed by the strict de dicto reading of (6), as well as to those expressed by (1) and (2) (if any), with different objects viz. with two tokens of the individual Cicero.

\subsection{Infinite regress with semantic contents?}

I already pointed out that, for Fine, positive or negative coordination among names shows up as positive or negative coordination among the objects to which they correspond (viz. occurrences of individuals, of properties, of relations within Russellian propositions). Could coordination also hold among occurrences of the same token object within token propositions? There seems to be no specific contraindication to that. Thus, we could say that e.g. positive coordination between 'Cicero' and 'Cicero' in (4) shows up as positive coordination not only between the occurrences of Cicero in (4p*) but also between the two occurrences of the very same token of Cicero contained in the token proposition that Cicero admires Cicero.

Now, using the notion of coordination among occurrences of the same token individual, we might introduce a fourth level of content: if primary, secondary and tertiary content are respectively Russellian propositions, coordinated propositions and token propositions, the quaternary content will be a coordinated token proposition, i.e. a structured proposition formed by the tertiary content (token proposition) plus coordinative links among its constituents. For example, the quaternary content of (4) will be the positively coordinated (token) proposition formed by the token proposition that Cicero admires Cicero plus the relation of positive coordination between the two occurrences of the token individual Cicero within it.

Even this is not the end of the story: exactly as tertiary content (token proposition) emerges from Russellian propositions plus coordinative links among their constituents, a quinary content will emerge from token propositions plus coordinative links among their constituents; this further content will be something like a second-order token proposition, i.e. a structured proposition composed of things identified by sets of positively coordinated occurrences of token objects. Proceeding in this way, token propositions of higher and higher order could be introduced. 
Now, if Fine is right in maintaining that a sentence has three contents-instead of only one, the primary content, as e.g. Salmon $(1986,1989)$ claims - I wonder why the number of contents assigned to the sentence should stop exactly at three instead of going on ad infinitum.

\subsection{True illogical belief?}

The SCC introduced in Sect. 4.1 for composite reports can easily be extended to a report of the form ' $S$ believes that $P a_{2} b_{2}$ ', e.g. (10), under the supposition that $S$ sincerely, on reflection and competently asserts a sentence of the form ' $P a_{1} b_{1}$ '.

(10) Tom believes that Cicero is not Tully.

Now, consider a piece of discourse containing the statements (1), (2) and (10), and suppose that Tom sincerely asserts "Cicero is not Tully". It could be shown that, under its strict de dicto reading, report (10) is correct, since the conditions (i)-(iii) (Sect. 4.1) are satisfied. The semantic content of this reading is the token proposition that Tom believes that Cicero is not Tully, which contains two distinct tokens of the individual Cicero: given the considered piece of discourse (and the corresponding body of expressed Russellian propositions), the token individual Cicero will be identified by the class of positively coordinated occurrences of Cicero including his occurrence in (1p) and his first occurrence in the Russellian proposition expressed by (10); the token individual Tully will be identified by the class of positively coordinated occurrences of Cicero including his occurrence in (2p) and his second occurrence in the Russellian proposition expressed by (10).

So, Tom believes, at the strict de dicto level, that one token individual Cicero (viz. the token Cicero) is not identical to another token of the same individual (viz. the token Tully). Since-as we have just seen-these two token individuals differ, Tom's belief (at the strict de dicto level) is true. This result clashes with the indisputable fact that the belief that Cicero is not Tully is false, impossible and illogical.

\section{Conclusion}

Fine's change of his original project from Russellianism enriched with coordination into a new sophisticated kind of Fregeanism involving token propositions does not really allow him to safeguard the principle of non-contradiction within his relational semantics; in addition, the notion of token proposition is problematic in some respects.

Actually, Fine could eliminate the true contradictions from his theory by taking Salmon's $(1986,1989)$ route and rejecting Negative Disquotation, to the effect that a report like (9) (which, at different levels, expresses self-contradictory propositions) would come out incorrect. On the other hand, both in his works and in face-toface conversations, Fine has manifested strong disinclination towards this proposal, which, according to him, introduces a counterintuitive "asymmetry" —on the one 
hand, e.g. Tom's sincere, on reflection and competent assertion of "Cicero is bald" (or of "I believe that Cicero is bald") suffices to ascribe to Tom the belief that Cicero is bald; on the other hand, his assertion (made under the same conditions) of "I do not believe that Tully is bald" does not authorize us to conclude that Tom does not believe that Tully is bald, contra Negative Disquotation.

It goes without saying that my doubts about semantic relationism do not take anything away from the extraordinary interest and value of Fine's theory.

Acknowledgments I am very grateful to Kit Fine, Kevin Mulligan and Marco Santambrogio for their insightful comments to this paper. I would also like to thank the participants at the 2012 New York Philosophy of Language Workshop for their helpful suggestions.

\section{References}

Braun, D., \& Saul, J. (2002). Simple sentences, substitutions, and mistaken evaluations. Philosophical Studies, 111, 1-41.

Fine, K. (2007). Semantic relationism. Oxford: Blackwell.

Fine, K. (2010). Comments on Scott Soames' 'Coordination problems'. Philosophy and Phenomenological Research, 81, 475-484.

Salmon, N. (1986). Frege's puzzle. Cambridge, MA: MIT Press/Bradford Books.

Salmon, N. (1989). Illogical belief. Philosophical Perspectives, 3, 243-285. 